\title{
Mach's Principle and the Laws of Logic
}

In this paper I wish to raise a philosophical question about logic, namely, the question whether its laws can consistently be thought of as analogous to those of the empirical sciences, i.e., as subject in some sense or other to test and confirmation, or whether, as is more often maintained, they must be thought of as analytic and a priori if not as coventional. In order to float the question, some general idea of what kind of activity logic is must be presupposed. The problem of logic I take to be as follows: Given the truth (or probability) of sentences $\{P\}$, what can we say (with what degree of confidence, etc.) about the truth (or probability) of sentences $\{Q\}$ ? The method of logic I take to consist in performing operations on the sentences $\{P\}$ or on supplementary sentences introduced for the purpose and in performing further operations on the sentences so generated, and so on until the sentences $\{Q\}$ or some obviously related sentences are generated. According to the rules employed in these operations we may then say that the sentences $\{Q\}$ are true or have a certain degree of probability in relation to the sentences $\{P\}$.

We thus arrive at a degree of confidence in the sentences $\{Q\}$. But what of our confidence in the whole procedure by which this degree of confidence is arrived at? Well, we can construct a second-order scheme for that and talk about the sentences $\{P\}$ and the rules by which we operated on them. We thus arrive at a degree of confidence in the procedure. But what of our confidence in this second-order scheme? And so on.

It is tacitly agreed by almost everyone except Quine that this regressive problem presents itself in two distinct cases. The first covers deductive inference and gives us absolute confidence in the conclusion on the object level as well as in the rules at all subsequent levels. The second covers everything else; we can't even think of pursuing the regression

AUTHOR'S NOTE: The original version of this paper was presented at a conference on induction held at the University of Minnesota in July 1968. 


\section{Peter Caws}

more than one or two levels, and even there we have to cut off debate by shifting attention from truth, probability, etc., to acceptability, epistemic utility, and the like. Whenever bits of the problem under its second aspect can be so arranged as to yield to the techniques proper to its first, this is instantly done; we thus have deductive theories of probability, utility, acceptance, and so on - a veritable deductivizing of all tractable parts of inductive logic. It seems reassuring to be able to say with deductive certainty that the conclusion follows inductively, even if we can't assert the conclusion itself with anything more than inductive probability. This activity takes place mainly on the first meta-level, and represents a kind of sidestepping of second- and higher-order inductive issues. My admiration for the people who do it is great and sincere, but I have no contribution to make along these lines. Instead I wish to confront one of the issues they sidestep and to suggest in a slightly different way from Quine's that the separation of cases will not stand up under scrutiny.

I start with Hume and in particular with the distinction between matters of fact and relations of ideas. We tend on the whole to give Hume too much credit as a contemporary and to make too little allowance for his belonging to the eighteenth century. Given the tenor of immediately preceding discussions, especially Berkeley's, Hume may certainly be excused for believing in "ideas," but that does not excuse us for following him in this aberration, whether or not we call things by the same name. The main contention of this paper is that matters of fact are enough - are, indeed, all we have - and that the complex matters of fact which we call "relations of ideas" (or which we call "logic," deductive or inductive) are reflections of the inclusive matter of fact which we call "the world" and are as contingent (or as necessary) as it is.

This contention can be looked upon as a philosophical analogue of the generalized form of Mach's principle. What Einstein called Mach's principle was of course the restricted claim that the inertia of a given body is determined by the distribution of matter in the physical universe. The generalized claim is that the laws of nature are so determined, and the philosophical analogue is that the laws of logic are determined, not to be sure by the distribution of matter, but by some feature or features of the world as a whole, so that they would be different if it were different. This means among other things at least a reinterpretation of what we 


\section{MACH'S PRINCIPLE AND THE LAWS OF LOGIC}

can mean by the expression "possible world," since as presently understood the limitations on possible worlds are precisely logical limitations, whereas if the world determines the logic (rather than the other way around) it would seem difficult to rule out a priori any world whatever, by any other argument, at least, than follows from the fact that this world already exists. Given logical rules (and the distinction between rules and laws is an important one in this context) we can of course explore the set of worlds in which they hold and the relative possibilities within this set; for that matter, we can devise systems with any rules we like, the only limitations being the scope of our imagination. (But that limitation is far from trivial.)

This, however, is running ahead somewhat. My proposal is really a double one, of which the analogical form of Mach's principle is only one element, the other element being the reconstruction of logic and all other relations of ideas as matters of fact. And even if both these elements were upheld, there would still remain the question of what light, if any, they threw on inductive logic and confirmation theory. The last question is the easiest. As I have already said, I claim to make no contribution to the technical discipline, but it may be that success in the philosophical enterprise would lead to a different view of what the technical discipline is all about and what can reasonably be expected of it. In particular it might help along the demystification of deductive logic, which as the unattainable paradigm has been responsible for driving so many inductive logicians to despair.

"Relations of ideas" are represented by Hume (for example, in the passage about consigning books to the flames) as the kinds of thing that form the object of reasoning about quantity or number. Matters of fact, on the other hand, are the kinds of thing that enter into causal relations. We can know some relations of ideas certainly, because (to use post-Humean language) they are analytic, definitional, and so on, and hence, as later came to be thought, empty of factual content. We can never know any matters of fact certainly, because in order to do so we would need certainty in causal relations, and these run into the notorious difficulty that future exceptions are always possible. (I include here of course future knowledge as well as future events.) I do not wish to rehearse all this, but to comment on the insertion of temporal considerations into the inductive case when they are absent from the deductive case. Suppose we were to ask, How do you know the deductive relations 


\section{Peter Caws}

will hold in the future any more than the inductive ones?

After a bit of spluttering, and assuming that the question is taken seriously (as I mean it to be), the reply to this is likely to be a challenge: How could the deductive relations be different, since they are rules of procedure and not laws of nature? What would it be like for them to be different? What conceivable test could be proposed that would reveal exceptions to them? To which I readily answer that I have no idea, I can't imagine an exception, it doesn't even make sense to me - but nor do I conclude, from these limitations on my powers of fantasy, that the thing is impossible. I can readily think of exceptions in the case of induction; I can't conceive of them in the case of deduction - but there must surely be more to it than that, or if there isn't, then the debate is shifting to my territory. For imagining, conceiving, and the like aren't logical categories at all, but rather psychological and thus empirical ones.

But for counterinstances to arise in the deductive case would surely involve a self-contradiction. Here the deductive logician is likely to start writing things down, showing that the very rules of the language $L$ entail the analyticity of this proposition or that. Now, however, I can afford to be generous. I have no objection whatever to analyticity within languages - for incorporating the law of contradiction as a rule in such languages; indeed, given the world we live in, it seems a sound move. But I can quite easily imagine a language in which the law of contradiction was not a rule, in which (let us say) truth-values were assigned at random; it might be spoken by some latter-day Epimenides, who would explain that Cretans aren't really liars, it's just that they don't.care one way or the other, and if they contradict themselves then they contradict themselves and that's all there is to it. We care about truth - and that is a fact, not a principle of logic. And it is our caring about truth, not just about the rules of the language, that makes us choose the rules we do.

If during the argument the logician has written something down, that is particularly gratifying. For inscriptions have a factual presence, and as long as we hang on to them we can keep ideas (and propositions and a lot of other metaphysical baggage) at arm's length. The curious dependence of logicians on factual things like inscriptions (not to mention brains) ought to tip us off to a discrepancy between theory and practice. In theory logic reaches for immutable truth (most logicians 
are at heart Platonists); in practice the logician writes something down on paper or a blackboard, thinks for a bit, imagines, conceives, etc., and then writes something else down. But, you say, these are mere tokens tokens stand for types, and the types refer to all those logical categories, truths, relations, and the like. That may be, but all I see are the inscriptions, the furrowed brow, the puzzled look. If I could give an adequate account of logic in terms of them, would we need the rest of the apparatus?

The kinds of matter of fact required for this reconstruction are two: first, obvious things like inscriptions and utterances, which can be located in the world easily enough alongside chairs and tables; second, a rather specialized kind of animal behavior (in this case the behavior of logicians) which can be located alongside eating and sitting, etc. I am prepared in the latter case to talk dispositionally about "abilities" provided it is understood that the possession of the ability in question is a factual matter to be judged in terms of behavior. What abilities does the logician need in order to ply his trade? They are not I think especially exotic abilities. The fundamental operation of logic is one that every functioning human being is capable of performing, indeed one that we all do perform all the time; I call it, borrowing from the classical English grammarians, "apposition," and it consists of taking two things - any two things - and putting (and holding) them together. I say "taking" and "putting" metaphorically; knowing a person's name is a case of apposition, so is knowing the French for an English word, so is using a metaphor, and so on. Apposition is a perfectly general binary operation, unconstrained by category considerations, and by means of it we build our world. The special behavior of logicians lies in the invention and following of special rules of apposition, which impose constraints under which we do not ordinarily work. (The laws of logic are not the laws of thought, any more than the laws of chess are the laws of moving objects on checkered surfaces. If Ayer or Berlin or Popper have blackand-white tiled bathrooms, that does not compel them to walk one square forward and one diagonally.)

The special rules of logic are formation-rules that preserve or at least safeguard type homogeneity and transformation-rules that preserve or at least keep track of truth. The chief talent that logic requires is an ability to stick to these rules; the looseness and redundancy, the ellipses and shortcuts of ordinary language give way to a more or less rigorous formal- 


\section{Peter Caws}

ism (which has nothing to do with notation). This talent is rarer than might be supposed, which accounts for the fact that logic (as I have pointed out elsewhere $)^{1}$ is too simple for many people who look for subtlety in its elements. It rapidly gets complex, of course, but its complexities always break down into simple elements as the fuzzy complexities of everyday life do not. People are always putting more or less complicated objects and expressions in apposition with each other and one another; this activity is governed by no principles other than immediate utility or intelligibility and the conventions of ordinary language and behavior, and consequently the coherence and even relevance of any element of the resulting structure with or to any other element are not guaranteed, indeed it is normal for this structure to be incoherent and fragmented. These defects do not often appear because different parts of the structure are usually brought into play in fairly specific contexts which not not overlap; when incompatible parts of it are activated at the same time the various psychological phenomena of dissonance are observed, and there are also contexts which activate no parts because they are just not comprehended. (Note that these structures are specific to individuals.) Logical structures on the other hand are such that except under Russellian or Gödelian stress all their parts are mutually consistent and no parts are lacking. The very idea of such comprehensive austerity is well-nigh inconceivable to the ordinary talker or thinker in daily life.

But what the logician does is different only in degree from what the ordinary man does, and it too is governed in the end by utility and intelligibility and convention. What the additional constraints of logic make possible are just those logical properties that we think of as characteristic, namely, analytic precision and synthetic open-endedness. Everyday thought is at once grosser and more limited than logical inference: grosser because it works with unanalyzed complex wholes, more limited because these cohere imperfectly with one another and relate for the most part intransitively, so that inferential sequences are always short. Still it is adequate to the conditions of its world and survives because of this adequacy. Its world is an aspect of the world; the temptation we have to resist is that of supposing that because logical refinements enable us to transcend the limitations of everyday thought

${ }^{1}$ Peter Caws, “ “. . Quine/Is Just Fine," " Partisan Review, 34, no. 2 (Spring $1967), 302$. 
they also enable us to transcend the actuality of the world. The logical operations we are capable of performing are just some of the things that our evolutionary development has equipped us to do, and like other features of our heredity they can be assumed to reflect a close adjustment to the facts of our environment. If these facts are contingent, then logical laws are also contingent, while if they are necessary the necessity of those laws is still extrinsic to logic and depends on an empirical relationship between it and the world.

In this light we need to look again at the doctrine that tautologies are empirically empty, perhaps to abandon it in favor of the doctrine that on the contrary they are empirically full, if I may so put it. ${ }^{2}$ Identity and contradiction aren't just logical rules (although they are that), nor are they laws of thought; they are laws of nature, reflecting just those properties of the world which, according to the analogous form of Mach's principle, determine the logic we are bound to use in dealing with it. They aren't obviously falsifiable (as remarked earlier, our imaginations aren't up to envisaging counterinstances, and by now it should be obvious why not) but their nonfalsifiability is clearly of a different kind from the nonfalsifiability of pseudoscientific or metaphysical claims. For that matter, in an important sense the other inductively established laws of nature aren't falsifiable either. It is one thing to know (to be able to imagine, etc.) what a counterinstance would be like, another to be able to produce one. We can of course construct interesting but as yet useless systems incorporating alternative rules, but we can do that for logic too. Physical laws, like logical ones, function as rules within the theoretical systems to which they belong and acquire the status of laws only on the successful application of those systems to empirical problems.

What holds for the laws of deductive logic holds equally and under precisely the same conditions for the laws of inductive logic. In neither case are we really guaranteed success in advance; if in the deductive case it is claimed that consistency itself requires the outcome, it must be recognized that consistency in language is one thing, consistency in the world another, and that the former, again, reflects the latter. We count heavily on the world's consistency and are perpetually vindicated in

\footnotetext{
${ }^{2}$ See in this connection Charles Hartshorne, "Some Empty Though Important Truths," in Sidney Hook, ed., American Philosophers at Work (New York: Criterion Books, 1956), pp. $225 \mathrm{ff}$.
} 


\section{Peter Caws}

doing so. We count heavily also on the world's continuity, on its regularity, and so on, and are vindicated in this too. The really serious difference between the cases lies in the information available in the premises, and from this point of view the current tendency to deductivize inductive problems seems to me entirely appropriate. But here a different distinction emerges, a form of the old distinction between theory confirmation and instance confirmation. In its traditional form the problem of induction focused mainly on scientific laws, which as conclusions of inductive inferences are regularly detached without modal qualifications and used as assertoric premises in making predictions. It would be absurd to say, every time we wanted to calculate quantities of chemical reagents or stresses in airframes, for example, "Well, the law is only confirmed to such and such a degree, so we can't really be sure how much we need," just as it would be absurd to place bets on the truth of special relativity theory. On the other hand in Bayesian estimation problems, statistical computations, etc., there isn't the same need for detachment; we can always go back to the original probabilities or the original figures and start again, and the probability estimate in the conclusion is of course the essential part of it. This difference in the use of inductive inference seems to me crucial. It is only in the former case that my version of Mach's principle can be thought of as applying, since in the latter our problem isn't with the behavior of the world exactly, but with the changing scope of our knowledge of it. The reason why the next raven should be black is quite different from the reason why the next ball drawn from the urn should be black.

I conclude by reiterating one or two points. Logic, like science, is a rule-governed human activity which consists in putting things (inscriptions, acts of judgment) in apposition with one another, in spatial juxtaposition, or in temporal sequence. Nobody can compel us to accept its conclusions, as the tortoise taught Achilles, but accepting (and detaching) them is one of the things it is useful to do in our dealings with the world. (Doing logic for no ulterior purpose is a form of having dealings with the world.) Within the systems we construct we can be as formal, as analytic, etc., as we like, but the choice among systems for use in dealing with the world rests, in the deductive as in the inductive case, on empirical (and in the long run on pragmatic) grounds. And there are limitations on the kinds of system we can construct, imposed by the finite scope of our intellect and its prior adaptation to the special 
circumstances in which we find ourselves. Success in theory, logical or scientific, consists in bringing it into parallel with the world so that it reflects essential features of the world. Some parallels are long-established and practically unquestioned, others are more recent and tentative. Also we can construct systems independently of questions of relevance, but their rules remain merely rules and are not to be confused with logical or empirical laws. 